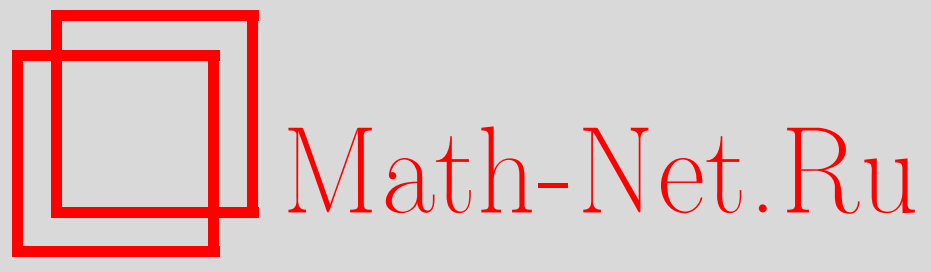

С. Тажетдинов, О специальных конгруэнц-подгруппах симплектических групп, Матем. заметки, 2006, том 80, выпуск 5, 770-772

DOI: https://doi.org/10.4213/mzm3086

Использование Общероссийского математического портала Math-Net.Ru подразумевает, что вы прочитали и согласны с пользовательским соглашением http://www . mathnet.ru/rus/agreement

Параметры загрузки:

IP : 34.229 .45 .116

26 апреля 2023 г., 16:12:03

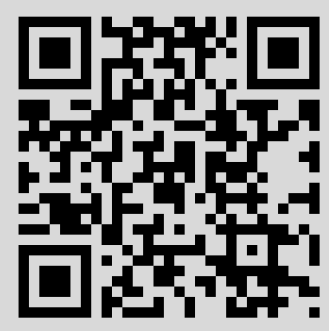




\section{О СПЕЦИАЛЬНЫХ КОНГРУЭНЦ-ПОДГРУППАХ СИМПЛЕКТИЧЕСКИХ ГРУПП}

\section{С. Тажетдинов}

Доказывается, что каждая специальная конгруэнц-подгруппа $S S p(V, I)$ симплектической группы $S p(V(R))$, где $R$ - кольцо стабильного ранга 1 с обратимым элементом 2 и $\operatorname{dim} V(R) \geqslant 4$, порождается содержащимися в ней симплектическими трансвекциями. Этот результат используется для полного описания нормальных подгрупп группы $S p(V(R))$.

Библиография: 8 названий.

Коммутативное кольцо $R$ называется кольцом стабильного ранга 1 , если в нем каждое равенство $\mathrm{id}(a, b)=R$, где $a, b \in R$, влечет за собой существование такого элемента $\alpha \in R$, что $a+\alpha b$ обратим в $R$. Примерами колец стабильного ранга 1 являются локальные и полулокальные кольца, кольца, регулярные по Нейману, и кольца размерности 0 (см. [1]).

Пусть $R$ - кольцо стабильного ранга 1 с обратимым элементом 2 и $V=V(R)-$ симплектическое пространство над $R$ размерности $r=2 m, m \geqslant 2$, т.е. свободный $R$-модуль с $r$ свободными порождающими и заданной на нем невырожденной кососимметрической билинейной формой $\beta(x, y)$. Группа автоморфизмов $\rho$ пространства $V$, оставляющих форму $\beta(x, y)$ инвариантной, т.е. удовлетворяющих соотношению $\beta(x \rho, y \rho)=\beta(x, y)$ при всех $x, y \in V$, называется симплектической группой над $V$ и обозначается $S p(V)$.

Пусть $I$ - идеал кольца $R$, отличный от $R$. Гомоморфизм колец $\lambda_{I}: R \rightarrow R I$ индуцирует гомоморфизм симплектических пространств (мы сохраняем обозначение)

$$
\lambda_{I}: V(R) \rightarrow V(R / I) .
$$

Пространство $V(R / I)$ образовано векторами $x \lambda_{I}, x \in V(R)$, причем форма $\beta\left(x \lambda_{I}\right.$, $\left.y \lambda_{I}\right)$ задается как $\beta(x, y) \lambda_{I}$. Считая $V(R / R)$ 0-модулем, распространим (1) и на случай $I=R$. Гомоморфизм (1) индуцирует сюръективный (см. [1]) гомоморфизм симплектических групп $\pi_{I}: S p(V(R)) \rightarrow S p(V(R / I))$, при котором $\lambda_{I}\left(\rho \pi_{I}\right)=\rho \lambda_{I}$ для $\rho \in S p(V(R))$. Здесь $S S p(V(R / R))$ означает единичную группу.

Пусть теперь $I$ - произвольный идеал кольца $R$. Общая и специальная конгруэнцподгруппы $G S p(V, I)$ и $S S p(V, I)$ определяются следующим образом:

$$
\begin{aligned}
& G S p(V, I)=(\text { center } S p(V(R / I))) \pi_{I}^{-1}, \\
& S S p(V, I)=(1) \pi_{I}^{-1}=\operatorname{Ker} \pi_{1} .
\end{aligned}
$$

(C) С. ТАЖетдинов, 2006 
Заметим, что $G S p(V, R)=S S p(V, R)=S p(V), G S p(V, 0)=\operatorname{center} S p(V), S S p(V, 0)$ $=\{1\}$.

Для $x \in V$ определим $\mathrm{id}(x)$ как наименьший идеал $J$ со свойством $x \lambda_{I}=0$, а для $\rho \in S p(V)$ определим вес $J(p)$ как наименьший идеал $J$ со свойством $\rho \in G S p(V, J)$. Если $H$ - подгруппа, то по определению положим $J(H)=\sum_{\rho \in H} J(\rho)$.

Далее, вектор $u \in V$ называется унимодулярным, если $\operatorname{id}(u)=R$. Если $u-$ унимодулярный вектор и $\alpha \in R$, то элемент $\tau_{u, \alpha} \in S p(V)$ вида $x \tau_{u, \alpha}=x+\alpha \beta(u, x) u$ называется симплектической трансвекцией. Если $u$ - унимодулярный вектор и $x \in V, \beta(u, x)=0$, то элемент $\sigma_{u, x} \in S p(V)$ вида

$$
y \sigma_{u, x}=y+\beta(u, y) x+\beta(x, y) u
$$

называется унитарной трансвекиией.

Для идеала $I$ кольца $R$ определим подгруппу

$$
E(V, I)=\operatorname{gr}\left(\tau_{u, \alpha} \mid u \in V, \operatorname{id}(u)=R, \alpha \in I\right) .
$$

В настоящей заметке мы покажем, что каждая специальная конгруэнц-подгруппа $S S p(V, I)$ порождается содержащимися в ней симплектическими трансвекциями (теорема 1). Этот результат, который имеет и самостоятельный интерес, используется для получения полного описания нормальных подгрупп группы $S p(V)$ (теорема 2). Итак, справедлива следующая

Теорема 1. Пусть $R$ - кольцо стабилъного ранга 1 с обратимым элементом 2 $u \operatorname{dim}(V(R)) \geqslant 4$. Тогда $S S p(V, I)=E(V, I)$ для каждого идеала I кольца $R$.

ДокАЗАТЕЛЬСтво. Включение $E(V, I) \leqslant S S p(V, I)$ очевидно, поскольку если $\alpha \in I$, то $\tau_{u, \alpha} \in S S p(V, I)$. Пусть теперь $\varphi \in S S p(V, I)$. Докажем, что $\varphi \in E(V, I)$. Из теоремы $5.6(\mathrm{~d})$ работы [2] легко следует, что $\varphi=\psi^{-1} \theta \psi$, где $\psi \in S p(V)$ и $\theta$ является произведением унитарных трансвекций вида $\sigma_{u, x}, \operatorname{id}(x) \in I$ (элемент $E(u, x)$ в работе [2] совпадает с $\left.\sigma_{u,-x}\right)$. Далее, повторяя ход рассуждений работы [3; c. $193-$ 197], получаем, что, в свою очередь, каждая унитарная трансвекция $\sigma_{u, x}$ является произведением симплектических трансвекций $\tau_{u, \alpha}$, причем если $\operatorname{id}(x) \subseteq I$, то $\alpha \in I$ для всех множителей $\tau_{u, \alpha}$. Поэтому достаточно показать, что $\psi^{-1} \tau_{u, \alpha} \psi \in E(V, I)$ для всех $\alpha \in I$, но это вытекает из известного тождества $\psi^{-1} \tau_{u, \alpha} \psi=\tau_{u, \psi, \alpha}$. Теорема доказана.

Эта теорема оказывается недостающим звеном в процессе полного описания нормальных подгрупп группы $S p(V(R))$. А именно, следствие из теоремы работы [4] с учетом только что доказанной теоремы дает возможность придать описаниям нормальных подгрупп группы $S p(V(R))$ следующую окончательную форму.

ТеОрема 2. Пусть $R$ - кольио стабилъного ранга 1 с обратимым элементом 2 $u \operatorname{dim} V(R) \geqslant 4$. Подгруппа $H$ нормалъна в группе $S p(V)$ тогда и только тогда, когда

$$
S S p(V, J) \leqslant H \leqslant G S p(V, J)
$$

для некоторого однозначно определенного идеала $J$ (совпадающего, следовательно, c $J(H))$. 
Из теорем, являющихся частными случаями этой теоремы, отметим только теорему 4.5 работы [2], в которой аналогичное описание получено другими методами при дополнительном предположении обратимости элемента 3 в кольце $R$. Теперь это ограничение оказывается лишним.

Пользуясь случаем, отметим, что в работах [5], [6] даны описания субнормальных подгрупп группы $S p(V)$ над некоторыми кольцами стабильного ранга 1, совпадающие для нормальных подгрупп с данным здесь описанием.

Отметим также, что при $\operatorname{dim} V(R)=2$ группа $S p(V)$ совпадает со специальной линейной группой $S L_{2}(V)$, и для этой группы исчерпывающие описания нормальных подгрупп даны в работах [7], [8].

\section{СПИСОК ЦИТИРОВАННОЙ ЛИТЕРАТУРЫ}

[1] B. Kirkwood, B. R. McDonald, "The symplectic group over a ring with one in its stable range", Pacific J. Math., 92:1 (1981), 111-125.

[2] H. Ishibashi, "Multiplicative and transitive symplectic groups", J. Algebra, 84 (1983), $115-127$.

[3] B. R. McDonald, Geometric Algebra over Local Rings, Marcel Dekker, New York-Basel, 1976.

[4] С. Тажетдинов, "Нормальное строение симплектических групп над кольцами стабильного ранга 1", Матем. заметки, 39:4 (1986), 512-517.

[5] С. Тажетдинов, "Субнормальное строение симплектических групп над локальными кольцами", Матем. заметки, 37:2 (1985), 289-298.

[6] С. Тажетдинов, "Субнормальное строение симплектических групп над $(2,3)$-полными кольцами”, Сиб. матем. ж., 34:6 (1993), 165-169.

[7] D. L. Costa, G. E. Keller, "On the normal subgroups of $\mathrm{SL}(2, A)$ ", J. Pure Appl. Algebra, 53:3 (1988), 201-226.

[8] D. L. Costa, G. E. Keller, "The E(2,A) sections of SL(2, A)", Ann. of Math. (2), 134:1 (1991), 159-188.

\section{С. Тажетдинов}

Поступило

Каракалпакский государственный университет им. Бердаха

02.10 .2000 